\title{
The SPD project for spin physics studies at the NICA accelerator complex
}

\author{
R. Tsenov*† \\ Joint Institute for Nuclear Research, Dubna, Russia \\ E-mail: tsenovejinr.ru
}

\begin{abstract}
The status of the Spin Physics Detector (SPD) project at the NICA accelerator complex now under construction in the JINR, Dubna is given. The expected availability of high luminosity colliding beams of polarized protons and deuterons opens wide opportunities for nucleon spin physics studies. Possible measurements are discussed together with a concept for general purpose detector to study the interactions of polarized particles in collider mode. Anticipated detector design and status of the Conceptual Design Report being prepared is given.
\end{abstract}

23rd International Spin Physics Symposium - SPIN2018 -

10-14 September, 2018

Ferrara, Italy

\footnotetext{
*Speaker.

${ }^{\dagger}$ on behalf of the SPD project team
} 


\section{Physics case}

Since the famous "spin crisis" that began in 1987, the problem of the nucleon spin structure remains one of the most intriguing puzzles in the contemporary high-energy physics. The central component of this problem, attracting for many years enormous both theoretical and experimental efforts, is the problem how the spin of the nucleon is built up from spins and orbital momenta of its constituents. The searches brought up a concept of spin-dependent parton distribution functions (PDFs) of the nucleon.

At leading order (leading twist, LO) in perturbative QCD expansion one may distinguish three kinds of PDFs. Two of them are well-known structure functions measured in deep inelastic scattering (DIS) and other processes: $f_{1}^{a}(x)$ ( $a$ is a parton flavor, often suppressed) is the density of unpolarized partons with longitudinal momentum fraction $x$ in an unpolarized nucleon, and $g_{1}(x)$ giving the net helicity of partons in a longitudinally polarized nucleon. The third one, the transversity $h_{1}(x)$, describing the density of partons with polarization parallel to that of a transversely polarized nucleon minus the density of partons with antiparallel polarization, is chiral-odd and requires a quark helicity flip in the scattering that cannot be achieved in the hard subprocess. Other parts of the cross section have to be explored for that. They are either chiral-odd fragmentation functions (FF) (e.g. Collins fragmentation function $H_{1}^{\perp}(x)$ in semi- inclusive DIS (SIDIS)) or yet another transversity, that of the second polarized incident hadron.

However, in addition to the information on the longitudinal behaviour in momentum space along the direction in which the nucleon is moving, drawing a complete three-dimensional picture of the nucleon also requires knowledge of the transverse motion of the partons [1,2]. A full account of the orbital motion can be given in terms of transverse-momentum dependent parton distribution functions (TMDs). There are eight leading-twist TMDs: $f_{1}\left(x, p_{T}\right), f_{1}^{\perp}\left(x, p_{T}\right), g_{1 L}\left(x, p_{T}\right)$, $g_{1 T}\left(x, p_{T}\right), h_{1}\left(x, p_{T}\right), h_{1 L}^{\perp}\left(x, p_{T}\right), h_{1 T}^{\perp}\left(x, p_{T}\right)$ and $h_{1}^{\perp}\left(x, p_{T}\right)$ [3].

In order to be sensitive to the intrinsic transverse parton momenta, it is necessary to measure the transverse momenta of the produced hadrons in the final state, e.g., in processes like SIDIS, hadron production in $e^{+} e^{-}$annihilation [1] or the transverse momentum of the lepton pair in the Drell-Yan processes in hadron-hadron collisions. Here, factorization theorem proven at leading twist $[4,5,6,7]$ allows to get information about TMDs and FFs that describe the hadronization process of the hit quark into the detected hadrons.

By measuring the angular distribution of produced hadrons in SIDIS, or Drell-Yan pairs in hadron collisions, it is possible to obtain information about all the eight leading-twist TMDs in combinations with the two leading-twist FFs.

Study of the gluon structure of the nucleon is of fundamental importance for understanding of its internal structure as a whole. The unpolarized gluon content is relatively well-known while our knowledge of the polarized distributions (including TMDs) is limited.

Measurement of single transverse spin asymmetry $A_{N}^{\gamma}$ in prompt-photon production at high $p_{T}$ in polarized $\mathrm{p}-\mathrm{p}$ and $\mathrm{d}-\mathrm{d}$ collisions could provide information about the gluon Sivers function, which is the mostly unknown function at the moment [8]. Study of prompt-photon production at large transverse momenta with longitudinally polarized proton beams could provide access to the gluon polarization $\Delta g$ via measurement of the longitudinal double spin asymmetry $A_{L L}^{\gamma}$ [9]. In general, the investigation of processes with prompt photons, as well as charmonium production, is 
a proven way to enhance the available rather scarce experimental information on the gluon content of the nucleon.

There are two main hard processes for the production of direct photons: gluon Compton scattering, $g q(\bar{q}) \rightarrow \gamma q(\bar{q})$, which dominates and gives access to the gluon distributions in the nucleon, and quark-antiquark annihilation, $q \bar{q} \rightarrow \gamma g$. The contribution of the latter to the total cross section presumably does not exceed $20 \%$. A few fixed-target and collider experiments performed unpolarized measurements of the prompt-photon production differential cross section [10]. A certain tension between data of the fixed-target results, the collider ones and the theoretical expectations takes place. New precise measurements could clarify the issue.

The opportunity to have high luminosity collisions of polarized protons and deuterons at the NICA collider allows to study a great variety of spin and polarization dependent effects in the hadron-hadron collisions. They are graphically represented in Figure 1.

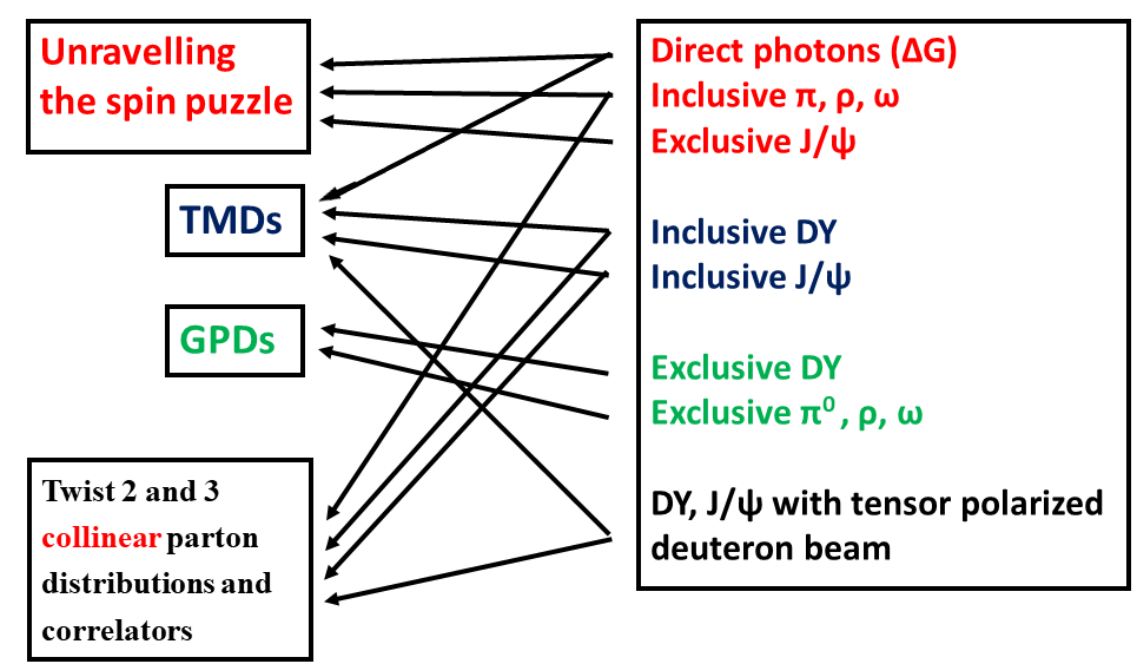

Figure 1: Graphical representation of the relations between the physics problems to be studied with SPD (left column) through measurements of reactions with various probes in the final state (right column). The arrows connect the measurements with the physics cases where they could contribute to.

\section{Polarized beams}

The NICA accelerator complex will deliver both longitudinally and transversely polarized beams at the SPD and MPD crossing points with overall polarization not less than $70 \%$ and lifetime comparable to that of the colliding beams themselves. A possibility to flip the spin of any bunch is also foreseen. Scan over the colliding energy with a step of $0.3-1.0 \mathrm{GeV}$ should be possible, as well as operation in asymmetric mode with respect to the particle momentum.

The main beam parameters are as follows: number of bunches $=22$, bunch length $\sigma=60$ $\mathrm{cm}$, orbit length $=503 \mathrm{~m}$, bunch velocity $v \approx 3 \times 10^{8} \mathrm{~m} / \mathrm{s}$, revolution time $\tau=1.67 \mu \mathrm{s}$, revolution frequency of a given bunch $=f \approx 0.6 \mathrm{MHz}$, time gap between bunches $\Delta \tau=76.0 \mathrm{~ns}$.

According to the calculations, luminosity of $1 \times 10^{30} \mathrm{~cm}^{-2} \mathrm{~s}^{-1}$ could be reached if each bunch is formed within one acceleration cycle of the Nuclotron ( $10^{11}$ polarized protons) and injected into 
the collider. In order to obtain $1 \times 10^{32} \mathrm{~cm}^{-2} \mathrm{~s}^{-1}$ each bunch should contain at least $10^{12}$ particles. A multi-bunch injection followed by a re-bunching in the collider rings might be needed for that. For details about the NICA project and polarized beams there see the talk by A. Kovalenko "Status and Perspectives of the NICA Project" at this conference.

\section{Spin physics detector (SPD)}

Design concept for a detector (Spin Physics Detector, SPD) capable to exploit the broad spin physics potential of the NICA collider is under preparation in the JINR. The physics program, requirements for the detector and the design ideas are based mainly on the Letter of Intent [11] submitted in 2014 and endorsed by the JINR Physics Advisory Committee (PAC) for Particle Physics. Further development and some new ideas can be found in the talks presented at the International Workshop on Spin Physics at NICA (Spin-Praha-2018) held 9-13 July 2018 in Prague, see http://spd.jinr.ru/spin-praha-2018/. The SPD facility is foreseen to be allocated in the south beam interaction point of the NICA collider.

The main requirements for the detector are listed below:

- close to $4 \pi$ geometry for secondary particles;

- high-precision $(\sim 50 \mu \mathrm{m})$ and fast vertex detector;

- high-precision $(\sim 100 \mu \mathrm{m})$ and fast tracking system;

- precision momentum measurement of secondary particles;

- good particle identification capabilities $\left(e^{ \pm}, \pi^{ \pm}, K^{ \pm}, \mu^{ \pm}, p^{ \pm}\right.$etc. $)$;

- good electromagnetic calorimeter;

- efficient muon range system;

- low material budget over the track paths;

- trigger and DAQ system able to cope with event rates at luminosity of $10^{32} \mathrm{~cm}^{-2} \mathrm{~s}^{-1}$;

- modularity and easy access to the detector elements in view of reconfiguration and further upgrade of the facility.

The aim is to have yet simple but universal detector that could be relatively easily reconfigured and/or upgraded. An idea about the composition and design of the SPD is presented in Figure 2.

The SPD length along the beam axis is $920 \mathrm{~cm}$, the diameter is $6.8 \mathrm{~m}$ and it consists of three parts: two end-caps and a barrel part. Each part has an individual magnet system: the end-caps - solenoidal coils, the barrel - toroidal magnetic system. The main detector systems, as shown in Figure 2, are as follows: Range System (RS) for muon identification, Electromagnetic Calorimeter (ECal), Particle Identification system (PID), Main tracker (TR) and Vertex Detector (also called Inner tracker, IT). The estimated total weight of the facility is of about 1800 tons. Such a design gives a possibility for upgrade and modification of each of the main detector systems and for performing measurements in different detector configurations. For example, for some of the physics tasks, e.g. detection of prompt photons, one could remove the toroidal magnet and the barrel tracker, thus decreasing significantly the material budget between the interaction point and the electromagnetic calorimeter.

A hybrid superconducting magnetic system is considered as a baseline. It consists of eight coils in the barrel section that generate a toroidal magnetic field in the central part of the detector and two pairs of two coils each in the end-caps that produce solenoidal-type magnetic field there. 

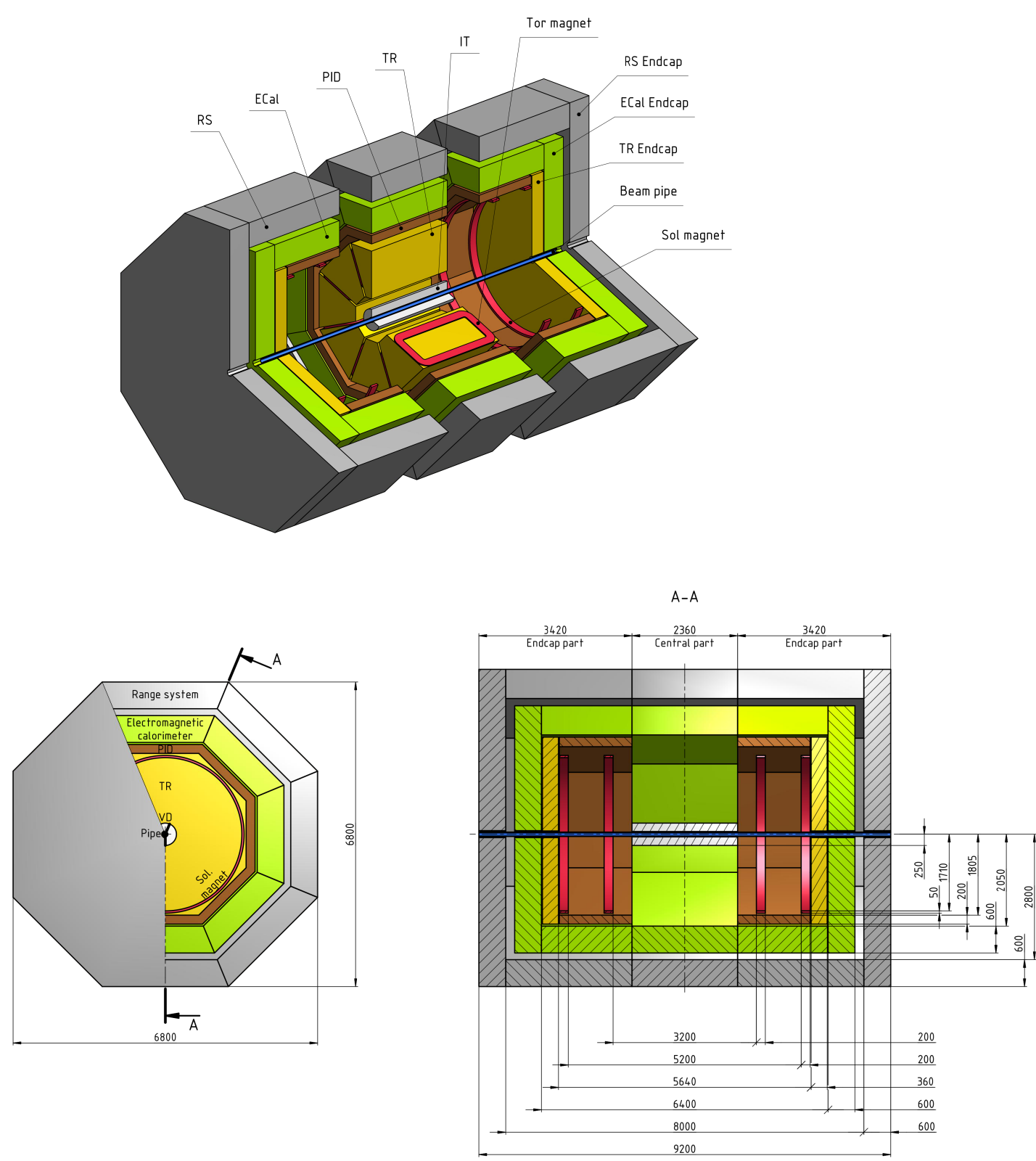

Figure 2: General view of the SPD detector (see text for details).

(see Fig. 3). Such a system minimizes the magnetic field on the beam axis, i.e. it does not change the polarization of the particles in the collision region and still creates strong enough field for momentum measurement.

\section{MC simulation and physics performance}

Monte Carlo simulation, event reconstruction for both simulated and real data, data analysis 

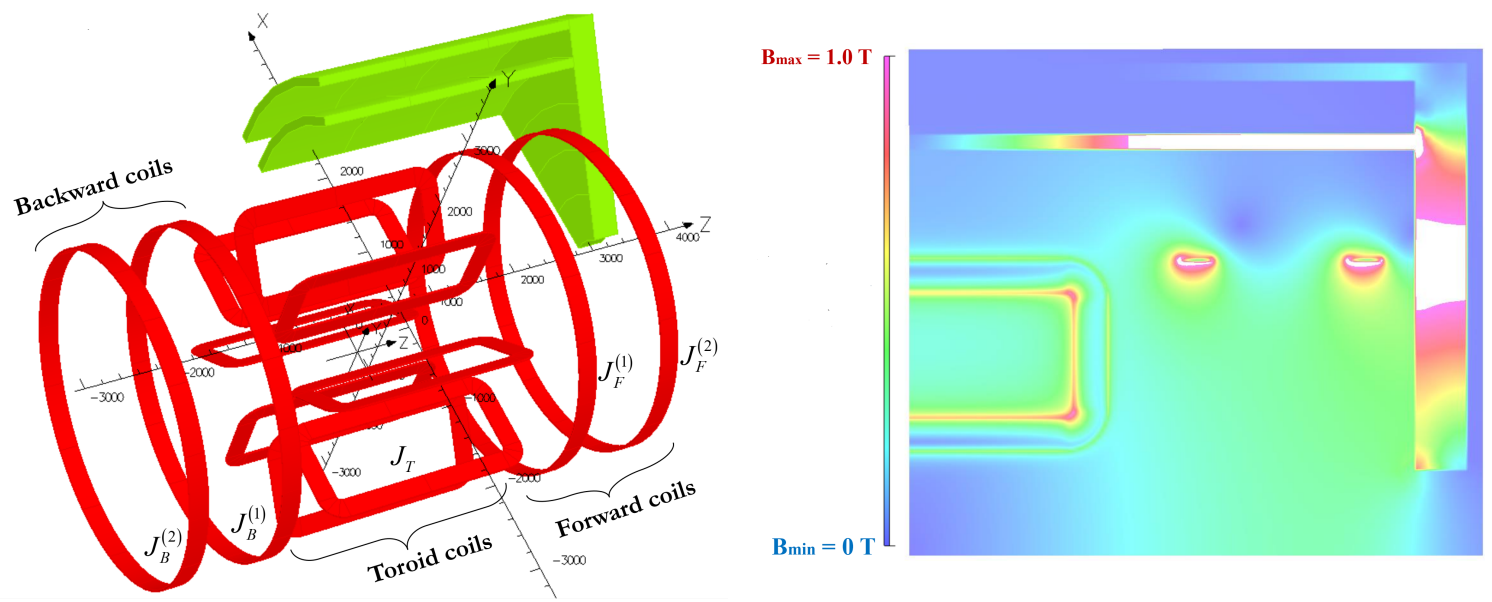

Figure 3: The hybrid magnetic system of the SPD (left). Calculation of the magnetic field strength in one quarter of the magnetized volume (right).

and visualization are to be coded within the object-oriented C++ toolkit SPDRoot. It is based on the FairRoot framework initially developed for the FAIR experiments at GSI Darmstadt and partially compatible with MPDRoot and BMNRoot software used for MPD and BM@N facilities at NICA. The detector description is based on the ROOT geometry approach, while the transport of the secondary particles through the material of the setup and simulation of the detector response is provided by GEANT4. The standard multipurpose generators like Pythia6 and Pythia8 as well as specialized generators can be used for simulation of primary nucleon-nucleon collision.

At this stage of the SPD conceptual design preparation a very preliminary simulations have been performed. For example, Drell-Yan pairs for two regions of the photon virtuality $Q^{2}$, i.e. the lepton pair invariant mass squared, $2<Q^{2}<8.5 \mathrm{GeV}^{2}$ and $Q^{2}>11 \mathrm{GeV}^{2}$, have been generated using a generator of polarized Drell-Yan pairs $[12,13]$. The statistical sample corresponds to $10^{5}$ Drell-Yan pairs, corresponding to of about 100 days $\left(10^{7} s\right)$ of data taking. Results for Sivers and Boer-Mulders asymmetries are shown in Figures 4 and 5, respectively. As it is seen, with statistics of $100 \mathrm{~K}$ events the unmeasured so far $x_{F}$ dependence of the respective asymmetries could be reconstructed.

Prompt photons are created mainly through Gluon Compton scattering (GCS) at SPD energies. At $\sqrt{s}=26 \mathrm{GeV}$ the corresponding cross section calculated in the leading order is $1.2 \mu \mathrm{b}$. Contribution of the competitive quark-antiquark annihilation process is an order of magnitude smaller. Unfortunately, the contribution of the decay photons dominates over the GCS signal in the whole kinematical region, but the relative fraction of the signal photons increases with increasing of the $p_{T}$. Rejection of photons from reconstructed $2 \gamma$ decays of $\pi^{0}$ and $\eta$-mesons and subsequent Monte Carlo-based statistical subtraction of the residual background is the only way to access the promptphoton production, presumably at high $p_{T}$. Figure 6a shows the $p_{T}$ distribution of the GCS photons vs. $x_{F}$. Estimated accuracy of the prompt-photon production asymmetries $A_{N}$ and $A_{L L}$ measurements is shown in Figure 6b. The data sample corresponds to $10^{7} \mathrm{~s}$ of data taking (about 100 days) with average luminosity $L=10^{32} \mathrm{~s}^{-1} \mathrm{~cm}^{-2}$. The inefficiency of the photon reconstruction is assumed to be due only to the geometrical acceptance and material distribution, no threshold for photon reconstruction in the ECal is applied. Leading order calculations are used for the GCS 

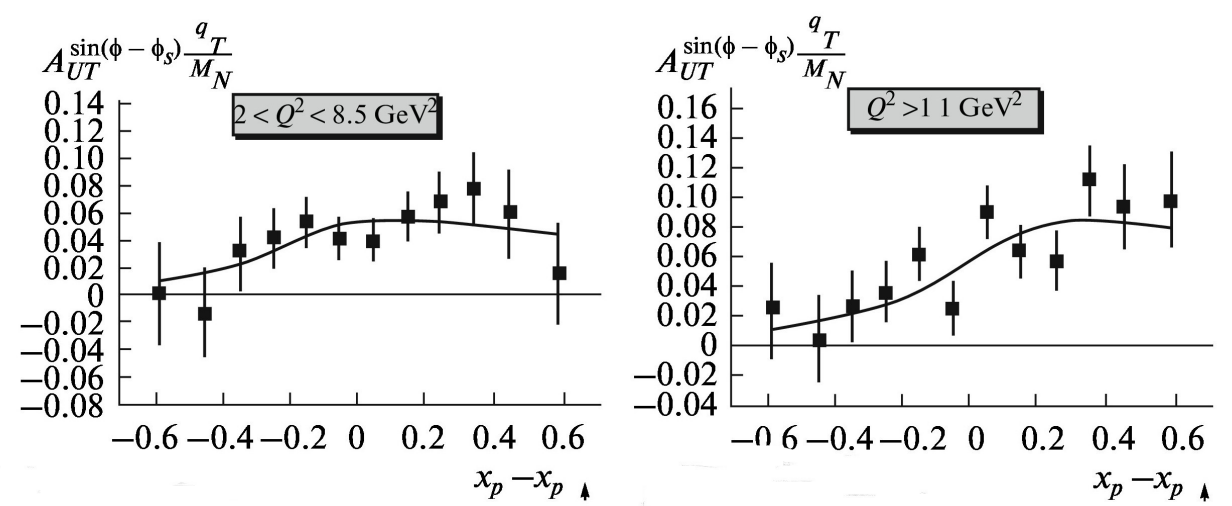

Figure 4: Simulated Sivers asymmetry of Drell-Yan pair production at NICA energies, $s=400 \mathrm{GeV}^{2}$ and in indicated intervals of photon virtuality $Q^{2}$. The function fitted to the MC data is taken from [14].
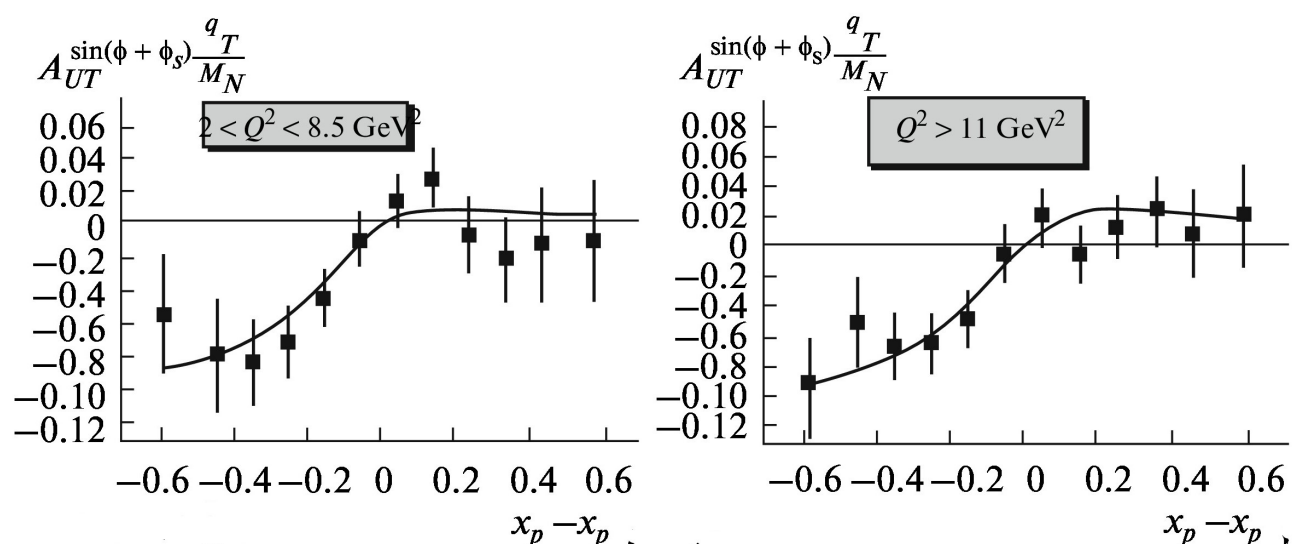

Figure 5: Simulated Boer-Mulders asymmetry of Drell-Yan pair production at NICA energies, $s=400 \mathrm{GeV}^{2}$ and in indicated intervals of photon virtuality $Q^{2}$.

cross-section. Only the $\pi^{0} \rightarrow \gamma \gamma$ decay is taken into account as a background. The average $\pi^{0}$ reconstruction efficiency is taken to be $75.3 \%$. The $p_{T}$ cut applied is $6 \mathrm{GeV} / c$ (see Figure 6a). The total number of the detected GCS photons with $p_{T}$ above this value is expected to be of about $6 \times 10^{6}$. It is seen that we could measure asymmetries as small as few percent.

The muon identification system of the SPD detector provides nice possibility to access the $J / \psi$ and $\psi^{\prime}$ signals via their decays into a dimuon pair. $\psi^{\prime}$ could also be accessed via the decay into $J / \psi \pi^{+} \pi^{-}$which has much higher branching fraction of $34.67 \%$. The charmonia states $\chi_{c 0,1,2}$ could be reconstructed, too, via detection in the ECal of the photon from the decay $\chi_{c 0,1,2} \rightarrow J / \psi \gamma$.

\section{Timeline and participation in the project}

We propose a three-year project for delivering of a technical design of the SPD facility based on the necessary simulation work, prototypes construction and test measurements. In 2019 a Con- 


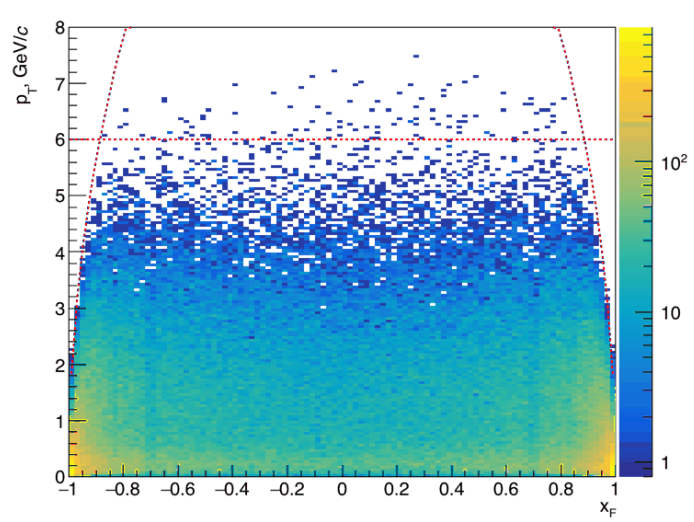

(a)

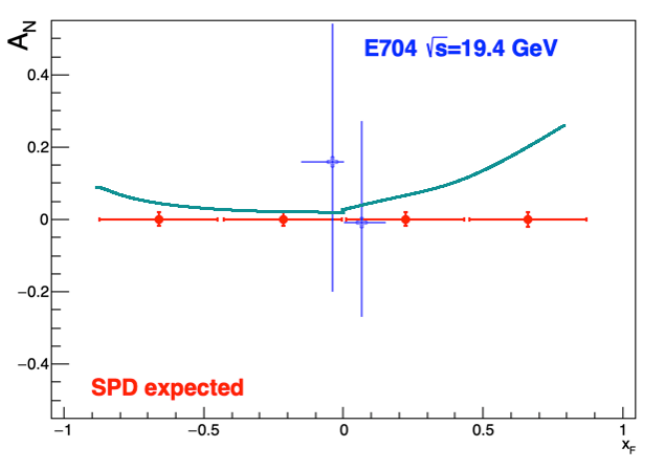

(b)

Figure 6: (a) $p_{T}$ distribution for GCS photons vs. $x_{F}$. (b) Expected accuracy of SPD measurements of $A_{N}$ and $A_{L L}$ as function of $x_{F}$. A previous measurement [15] in a similar kinematical range $-0.15<x_{F}<0.15$, $2.5 \mathrm{GeV} / c<p_{T}<3.1 \mathrm{GeV} / c$ is shown as well as theory predictions [16], [17].

ceptual Design Report (CDR) will be prepared and submitted to the PAC. In case of its approval the next two years will be devoted to prototyping and design work in order to be able to deliver a Technical Design Report (TDR) by the end of 2021. We hope to be able to start construction of the detector in 2022 and perform first measurements somewhere in 2025.

Host institute of the project and the one with largest participation so far is the Laboratory of High-Energy Physics of the Joint Institute for Nuclear Research. The Laboratory of Nuclear Problems is the other laboratory of the JINR that participates in the project with substantial number of researchers.

An international collaboration for preparation of CDR, TDR, construction of the facility and performing measurements with it is being set up. Up to now (January 2019) eighteen institutes from the JINR Member States and other countries have expressed their interest for participation in the project and indicate that they could allocate resources and manpower. We are keen to involve in the project more groups of researchers from all over the world that are willing to take part in this enterprise.

\section{Acknowledgments}

The speaker is deeply grateful to all colleagues in the SPD team that have supplied material for the conference presentation and this paper, as well as for the careful reading of the draft and providing comments.

\section{References}

[1] J. C. Collins, What exactly is a parton density?, Acta Phys. Polon. B34 (2003) 3103 [hep-ph/0304122].

[2] J. C. Collins, T. C. Rogers and A. M. Stasto, Fully unintegrated parton correlation functions and factorization in lowest-order hard scattering, Phys. Rev. D77 (2008) 085009 [0 708 . 2833 ]. 
[3] D. Boer and P. J. Mulders, Time reversal odd distribution functions in leptoproduction, Phys. Rev. D57 (1998) 5780 [hep-ph/9711485].

[4] J. C. Collins and D. E. Soper, Back-To-Back Jets in QCD, Nucl. Phys. B193 (1981) 381.

[5] J. C. Collins and A. Metz, Universality of soft and collinear factors in hard-scattering factorization, Phys. Rev. Lett. 93 (2004) 252001 [hep-ph/ 040824 9].

[6] X.-d. Ji, J.-p. Ma and F. Yuan, QCD factorization for semi-inclusive deep-inelastic scattering at low transverse momentum, Phys. Rev. D71 (2005) 034005 [hep-ph/ 0404183 ].

[7] X.-d. Ji, J.-P. Ma and F. Yuan, QCD factorization for spin-dependent cross sections in DIS and Drell-Yan processes at low transverse momentum, Phys. Lett. B597 (2004) 299 [hep-ph/0405085].

[8] D. Boer, C. Lorcé, C. Pisano and J. Zhou, The gluon Sivers distribution: status and future prospects, Adv. High Energy Phys. 2015 (2015) 371396 [1504 . 04332$].$

[9] H.-Y. Cheng and S.-N. Lai, Spin Asymmetry in Proton Proton Collisions as a Probe of Sea and Gluon Polarization in a Proton, Phys. Rev. D41 (1990) 91.

[10] P. Aurenche, M. Fontannaz, J.-P. Guillet, E. Pilon and M. Werlen, A New critical study of photon production in hadronic collisions, Phys. Rev. D73 (2006) 094007 [hep-ph/ 0602133 ].

[11] I. A. Savin et al., Spin Physics Experiments at NICA-SPD with polarized proton and deuteron beams, EPJ Web Conf. 85 (2015) 02039 [1408.3959].

[12] A. N. Sissakian, O. Yu. Shevchenko, A. P. Nagaitsev and O. N. Ivanov, Polarization effects in Drell-Yan processes, Phys. Part. Nucl. 41 (2010) 64.

[13] A. Sissakian, O. Shevchenko, A. Nagaytsev and O. Ivanov, Transversity and T-odd PDFs from Drell-Yan processes with p p, p D and D D collisions, Eur. Phys. J. C59 (2009) 659 [0807.2480].

[14] J. C. Collins, A. V. Efremov, K. Goeke, S. Menzel, A. Metz and P. Schweitzer, Sivers effect in semi-inclusive deeply inelastic scattering, Phys. Rev. D73 (2006) 014021 [hep-ph/ 0509076 ].

[15] E704 collaboration, Measurement of single spin asymmetry for direct photon production in $p p$ collisions at 200-GeV/c, Phys. Lett. B345 (1995) 569.

[16] N. Hammon, B. Ehrnsperger and A. Schaefer, Single-transverse spin asymmetry in prompt photon production, J. Phys. G24 (1998) 991.

[17] J.-w. Qiu and G. F. Sterman, Single transverse spin asymmetries in direct photon production, Nucl. Phys. B378 (1992) 52. 